\title{
Seroprevalencia de la enfermedad de Chagas en embarazadas del departamento de Cordillera antes y después de la implementación del control prenatal de Chagas en los periodos 1997 y 2011
}

\author{
Graciela Meza \\ Instituto Superior Profesional Avanzado, Asunción - Paraguay
} Cómo referenciar este artículo/
How to reference this article:

\begin{abstract}
Meza G. Seroprevalencia de la enfermedad de Chagas en embarazadas del departamento de Cordillera antes y después de la implementación del control prenatal de Chagas en los periodos 1997 y 2011. Mem. Inst. Investig. Cienc. Salud. 2016;14(3):73-80
\end{abstract}

\section{RES U M E N}

La enfermedad de Chagas es causada por el parásito Trypanosoma cruzi, afecta aproximadamente a 10 millones de individuos se encuentra presente en casi toda América Central y del Sur. En Paraguay se estima que existen aproximadamente 150.000 personas infectadas, de las cuales 61.000 son mujeres en edad fértil y que estarían naciendo alrededor de 600 niños infectados por transmisión congénita por año. El departamento de Cordillera es una zona endémica de la Región Oriental del país, donde la seroprevalencia en mujeres embarazadas en la década de los noventa era alrededor del $12 \%$. Este estudio descriptivo de corte transverso retrospectivo se planteó con el fin determinar la seroprevalencia de la enfermedad de Chagas en embarazadas del departamento de Cordillera que asistieron a su control prenatal en los años 1997 y 2011 . Se realizó el análisis de la base de datos de embarazadas del departamento de Cordillera. Se analizaron los resultados de la serología para $T$. cruzi de 7.898 embarazadas en 5 laboratorios de diagnóstico correspondiente a 20 distritos del departamento de Cordillera. Se encontró que la prevalencia de infección con $T$. cruzi en embarazadas en éste departamento en el año 1997 fue del $15 \%$ y en el 2011 del 6\%, lo que marca un descenso del 9\%. La información obtenida contribuye al sistema de control prenatal de la enfermedad en la III Región Sanitaria del departamento de Cordillera, porque demuestra la efectividad del sistema de diagnóstico prenatal y los métodos de prevención implementados por el Ministerio de Salud Pública y Bienestar Social.

Palabras clave: Enfermedad de Chagas, seroprevalencia, embarazadas.

\section{Seroprevalence of Chagas disease in pregnant women of the Cordillera department before and after the implementation of prenatal control of Chagas in the periods 1997 and 2011}

\section{A B S T R A C T}

Chagas disease is caused by the Trypanosoma cruzi parasite, affects approximately 10 millons individuals worldwide and is present in almost all Central and South America. In Paraguay, 150,000 people are estimated to be infected, 61,000 of them are women in reproductive age and about 600 congenitally infected children would be born per year. The Cordillera department is an endemic area of the Oriental Region of the country, where seroprevalence in pregnant women in the 90's was around $12 \%$. This retrospective transversal descriptive study was designed to determine the seroprevalence of Chagas disease in pregnant women of the Cordillera department that attended prenatal control during 1997 and 2011. Analysis of the database of pregnant women of the Cordillera Fecha de recepción: setiembre 2016. Fecha de aceptación: noviembre 2016 Autor correspondiente: Graciela Meza. Instituto Superior Profesional Avanzado, Asunción - Paraguay E-mail: graciemezaacosta@gmail.com 
department was carried out. Serology for $T$. cruzi of 7,898 pregnant women performed in 5 laboratories corresponding to 20 districts of the Cordillera was analyzed. The prevalence of T. cruzi infection in pregnant women of this department in the year 1997 was $15 \%$ and in 2011 it was $6 \%$, which indicates a reduction of $9 \%$. The collected information contributes to the prenatal control system of the disease in the III Sanitary Region of the Cordillera department, since it showed the efficiency of the prenatal diagnosis system and the methods of prevention implemented by the Ministry of Public Health.

Keyword: Chagas disease, seroprevalence, pregnant women.

\section{INTRODUCCIÓN}

La enfermedad de Chagas, también llamada tripanosomiasis americana, es una enfermedad potencialmente mortal causada por el parásito protozoo Trypanosoma cruzi. Esta enfermedad constituye uno de los principales problemas de salud pública en diversos países, principalmente en América Latina, y aproximadamente 10 millones de personas están infectadas por el $T$. cruzi y actualmente se ha propagado a otros continentes $(1,2)$.

Una de las formas de transmisión de la enfermedad es la congénita. La transmisión congénita ocurre en todas las regiones endémicas de América Latina y depende directamente de la infección en las mujeres en edad fértil, quienes han adquirido la infección con el $T$. cruzi mayormente por transmisión vectorial. Se estima que aproximadamente 15.000 infantes nacen infectados anualmente por transmisión vertical en América Latina, y que el número de mujeres seropositivas de 15 a 44 años es de alrededor de $1.809 .507(3,4)$. En Paraguay se estima que existen aproximadamente 150.000 personas infectadas, de las cuales 61.000 son mujeres en edad fértil y que estarían naciendo alrededor de 600 niños infectados por transmisión congénita por año (5). Está comprobado que el tratamiento es efectivo solo en etapas tempranas de la enfermedad, razón por la cual es muy importante realizar un diagnóstico temprano en niños nacidos de madres infectadas, para lo cual se necesita conocer la seroprevalencia de la enfermedad en embarazadas de regiones endémicas $(4,6)$.

La transmisión vectorial es una de las formas más importantes de transmisión de $T$. cruzi, en los países de la región, a través de los controles de vigilancias entomológicas se ha logrado la disminución de la transmisión por vectores $(7,8,9)$. En Paraguay el principal vector es el Triatoma insfestans conocido en el país como vinchuca o chicha guazú, que se encontraba ampliamente distribuido registrando rangos de prevalencias de infestación entre $11 \%$ y $60 \%$ para la región oriental, en el año 2003 , los índices de infestación domiciliar se registraron en rangos entre $0,1 \%$ y $20 \%$, que posteriormente, se logró reducir a valores de infestación de 0,1 a 1,5\% para la región oriental. Se logró la interrupción de la transmisión vectorial por $T$. infestans en esa región del país en el año 2008, certificada por la Comisión Intergubernamental de la Iniciativa Sub regional del Cono Sur (INCOSUR) $(10,11)$.

La enfermedad de Chagas está asociada con la pobreza y las malas condiciones de vivienda que favorecen la instalación del insecto vector; constituye un fenómeno complejo donde conviven situaciones biológicas, epidemiológicas, culturales, sociales, políticas y económicas por lo que el control del vector y el mejoramiento de la vivienda son aún los métodos más seguros para su prevención (8).

Las medidas de prevención y control tomadas en el marco de las Iniciativas Subregionales de Chagas, junto a circunstancias sociales, y económicas favorables en Latinoamérica, han marcado, en términos generales, descensos en la incidencia y prevalencia de la infección humana por T.cruzi. En el país como parte integrante de la Iniciativa del Cono Sur (INCOSUR) para la eliminación de $T$. infestans y control de la enfermedad de Chagas transfusional, se realizaron dos importantes actividades a nivel entomológico a partir del año 1999: una primera etapa de relevamiento entomológico y control químico como línea de base en todos los departamentos endémicos (1999 a 2005), y una segunda etapa con la introducción de sistemas de vigilancia entomológica implementada en los departamentos Cordillera, Paraguarí, Concepción, San Pedro y Guairá de la Región Oriental al año de las actividades de control químico $(5,10,12,13)$.

Cordillera es el tercer departamento del país, es una zona endémica de la Región Oriental del Paraguay, donde la seroprevalencia de la enfermedad de Chagas en embarazadas del grupo atareo de 15 a 45 años es del 10 al 12\% (14). Según estudiopublicado en el año 2009, la prevalencia de la trasmisión congénita en 12 años fue del 2 al 7\% (4). A nivel nacional los bancos de sangre registran una prevalencia de la enfermedad del $3,2 \%(3,12)$. Estudios recientes sobre la serología reactiva para $T$. cruzi en donantes de sangre de todo el 
país, demuestran que en el año 2011 el departamento de Cordillera presento una prevalencia del 5,91\%, siendo uno de los departamentos con frecuencia de casos reactivos mayor a la media nacional de 2,92\% (15). Por todo lo expuesto se planteó este estudio con el objetivo de conocer la seroprevalencia y la situación de la enfermedad de Chagas en embarazadas que asistieron a su control prenatal en los hospitales y centros de salud de los 20 distritos del departamento, en el año 1997; poco tiempo después de la implementación del programa de control prenatal de la enfermedad de Chagas que fue en julio de 1995 (16) y en el año 2011; después de la implementación del programa.

\section{MATERIALES Y MÉTODOS}

Diseño de estudio. Encuestas seriadas de datos secundarios.

Población de estudio. Gestantes que concurrieron a su control prenatal en los hospitales y centros de salud de todos los distritos del departamento de Cordillera en los años 1997 y 2011.

Fuente de datos: Mediante nota de pedido a la III Región Sanitaria del departamento de Cordillera, se accedió a la base de datos del Programa de Control Pre- Natal, de la que se obtuvo los datos de embarazadas que asistieron a su control prenatal en los hospitales y centros de salud de todos los distritos del departamento de Cordillera en los años 1997 y 2011. La población enfocada en el año 1997 fue un total de 5243 muestras de embarazadas y en el año 2011 de2655 muestras de embarazadas. El departamento de Cordillera dispone de cinco laboratorios de diagnóstico que son centros de referencia para los 20 distritos del departamento. Los laboratorios reciben las muestras remitidas y datos de las embarazadas para su procesamiento. Los cinco laboratorios y los distritos de los cuales reciben las muestras son:

Laboratorio de Caacupé: Caacupé, Piribebuy, Atyra, Altos, San Bernardino, Nueva Colombia, Loma Grande y Mbocayaty.

Laboratorio de Tobati: Tobatí

Laboratorio de Eusebio Ayala: Eusebio Ayala, Itacurubí de la Cordillera, Valenzuela, Santa Elena, Isla Pucú.

Laboratorio de Caraguatay: Caraguatay, San José Obrero, Primero de Marzo.

Laboratorio de Arroyos Esteros: Arroyos y Esteros, Emboscada, Juan de Mena.

\section{Método}

El análisis se realizó sobre los registros de resultados del Test de ELISA para Chagas en embarazadas que concurrieron para su control prenatal en los años 1997 y 2011. Cabe destacar que toda mujer seropositiva detectada durante el control prenatal no ingresa al tamizaje serológico en embarazos posteriores en los hospitales y centro de salud de la III Región Sanitaria del departamento de Cordillera, porque a las mismas se le provee de una tarjeta identificatoria de infección chagásica, además de registrar este dato en su ficha clínica. Con el análisis realizado se obtuvieron las frecuencias de embarazadas infectadas con T. cruzi en los años 1997 y 2011.

\section{Análisis estadístico}

Los datos fueron obtenidos en planillas impresas que posteriormente fueron introducidos en una hoja de cálculo de Microsoft Office Excel 2007 y analizada mediante EPI-INFO 7 (CDC, Atlanta). Las frecuencias fueron obtenidas en forma absoluta y porcentual. La comparación de las seroprevalencias entre los DOS periodos se hizo con la prueba chi cuadrado a un nivel de significancia de 0,05.

\section{RESULTADOS}

En el departamento de Cordillera se analizaron en el año 1997 un total de 5.243 muestras de embarazadas y se diagnosticaron 771 casos positivos para la enfermedad de Chagas (seroprevalencia: 15\%); en el año 2011 de las 2.655 muestras de embarazadas analizadas se diagnosticaron 168 casos positivos (seroprevalencia: 6\%), observándose un descenso altamente significativo (valor $\mathrm{p}<0,00001$ ) de $9 \%$. (Figura 1) 


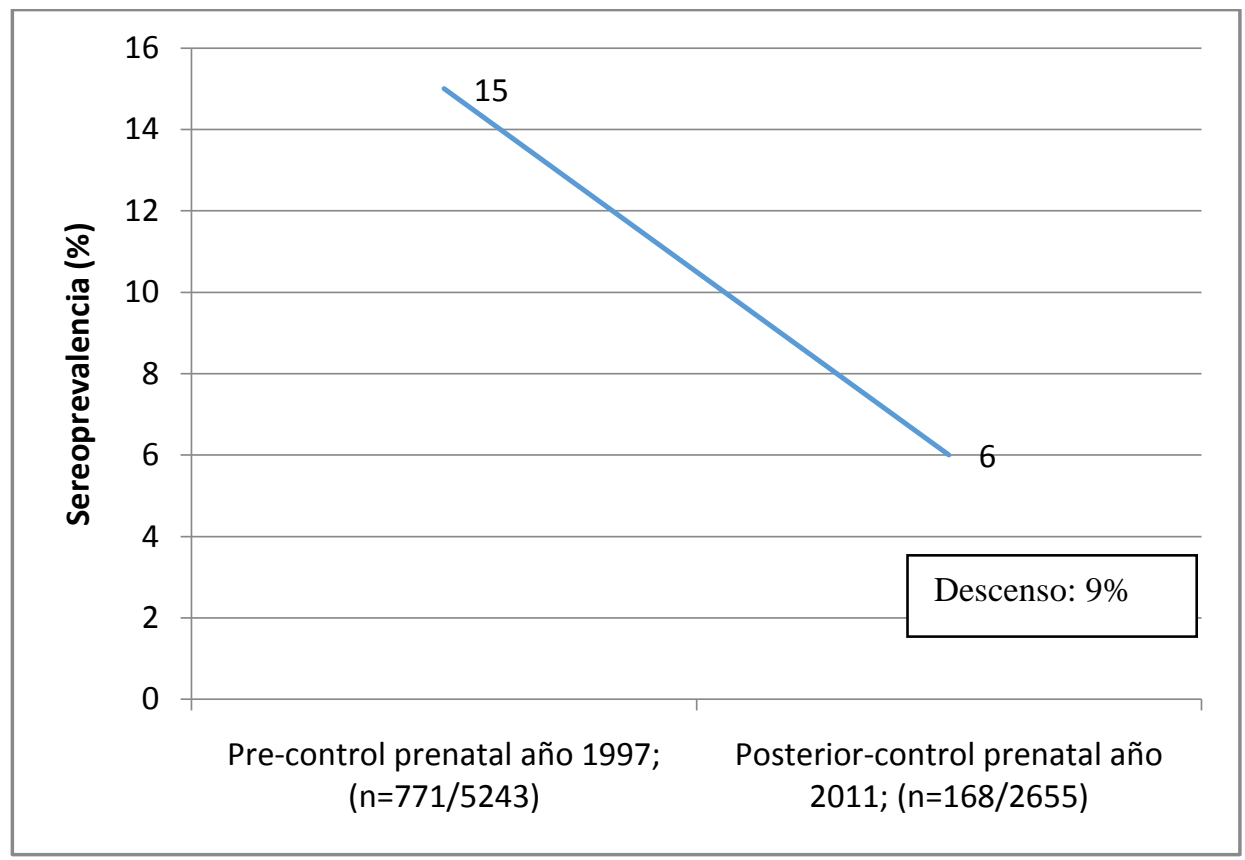

Fuente: Base de datos proveído por la III Región Sanitaria de Cordillera.

Figura 1. Seroprevalencia de la enfermedad de Chagas en los años de 1997 y 2011.

En la Tabla 1 y en la Figura 2 se comparan los dos periodos de estudio por distrito y por laboratorio de diagnóstico, observándose descensos en la prevalencia de la enfermedad de Chagas entre $1 \%$ en el distrito de Mbocayaty y $22 \%$ en San Bernardino. En general, el número de muestras procesadas se redujo en el 2011 y en el laboratorio de Emboscada no se procesó ninguna muestra.

Tabla 1. Comparación de la seroprevalencia de la enfermedad de Chagas en embarazadas de los 20 distritos del departamento de Cordillera entre los años 1997 y 2011

\begin{tabular}{|c|c|c|c|c|c|c|c|c|}
\hline \multirow[b]{2}{*}{$\begin{array}{l}\text { Laboratorio de } \\
\text { diagnóstico }\end{array}$} & \multirow[b]{2}{*}{ Distrito } & \multicolumn{3}{|c|}{ AÑO 1997} & \multicolumn{3}{|c|}{ AÑO 2011} & \multirow[b]{2}{*}{$\begin{array}{c}\text { Descenso de } \\
\text { prevalencia } \\
(\%)\end{array}$} \\
\hline & & $\begin{array}{c}\text { No } \\
\text { muestras } \\
\text { analizadas }\end{array}$ & $\begin{array}{l}\text { No } \\
\text { pos. }\end{array}$ & $\begin{array}{l}\text { Prev. } \\
(\%)\end{array}$ & $\begin{array}{c}\text { No } \\
\text { muestras } \\
\text { analizadas }\end{array}$ & No pos. & $\begin{array}{l}\text { Prev. } \\
(\%)\end{array}$ & \\
\hline \multirow{9}{*}{ Caacupé } & Caacupé & 1714 & 210 & 12 & 821 & 44 & 5 & 7 \\
\hline & Piribebuy & 480 & 85 & 18 & 150 & 6 & 4 & 16 \\
\hline & Altos & 191 & 55 & 29 & 186 & 21 & 11 & 18 \\
\hline & Atyra & 219 & 28 & 13 & 112 & 6 & 5 & 8 \\
\hline & San Bernardino & 266 & 66 & 25 & 32 & 1 & 3 & 22 \\
\hline & Loma Grande & 57 & 14 & 25 & 28 & 5 & 18 & 7 \\
\hline & Nueva & 53 & 14 & 26 & 50 & 5 & 10 & 16 \\
\hline & Colombia & & & & & & & \\
\hline & Mbocayaty & 42 & 5 & 12 & 27 & 3 & 11 & 1 \\
\hline \multirow{4}{*}{ Tobati } & Sub-total & 3022 & 477 & 16 & 1406 & 91 & 6 & 10 \\
\hline & Tobati & 459 & 70 & 15 & 362 & 23 & 6 & 9 \\
\hline & Eusebio Ayala & 375 & 45 & 12 & 206 & 12 & 6 & 6 \\
\hline & $\begin{array}{l}\text { Itacurubi de la } \\
\text { Cordillera }\end{array}$ & 200 & 16 & 8 & 62 & 4 & 6 & 2 \\
\hline \multirow{5}{*}{$\begin{array}{c}\text { Eusebio } \\
\text { Ayala }\end{array}$} & Valenzuela & 96 & 8 & 8 & 11 & 0 & 0 & 8 \\
\hline & Santa Elena & 113 & 18 & 16 & 12 & 0 & 0 & 16 \\
\hline & Isla Pucu & 153 & 23 & 15 & 12 & 0 & 0 & 15 \\
\hline & Sub-total & 937 & 110 & 12 & 303 & 16 & 5 & 7 \\
\hline & Caraguatay & 174 & 28 & 16 & 105 & 3 & 3 & 13 \\
\hline \multirow{4}{*}{ Caraguatay } & San José Obrero & 57 & 7 & 12 & 14 & 0 & 0 & 12 \\
\hline & Primero de Marzo & 87 & 12 & 14 & 29 & 1 & 3 & 11 \\
\hline & Sub-total & 318 & 47 & 15 & 148 & 4 & 3 & 12 \\
\hline & Arroyos y Esteros & 311 & 34 & 11 & 346 & 29 & 8 & 3 \\
\hline Arroyos y & Emboscada & 183 & 31 & 17 & 0 & 0 & 0 & - \\
\hline \multirow[t]{2}{*}{ Esteros } & Juan de Mena & 13 & 2 & 15 & 90 & 5 & 6 & 9 \\
\hline & Sub-total & 507 & 67 & 13 & 436 & 34 & 8 & 5 \\
\hline Total & & 5243 & 771 & $15 \%$ & 2655 & 168 & $6 \%$ & $9 \%$ \\
\hline
\end{tabular}

Fuente: Base de datos proveído por la III Región Sanitaria de Cordillera. Año 2012 
En la Figura 2 se muestra el descenso en la seroprevalencia de la enfermedad de Chagas en las embarazadas, por distritos del departamento de Cordillera en 15 años.

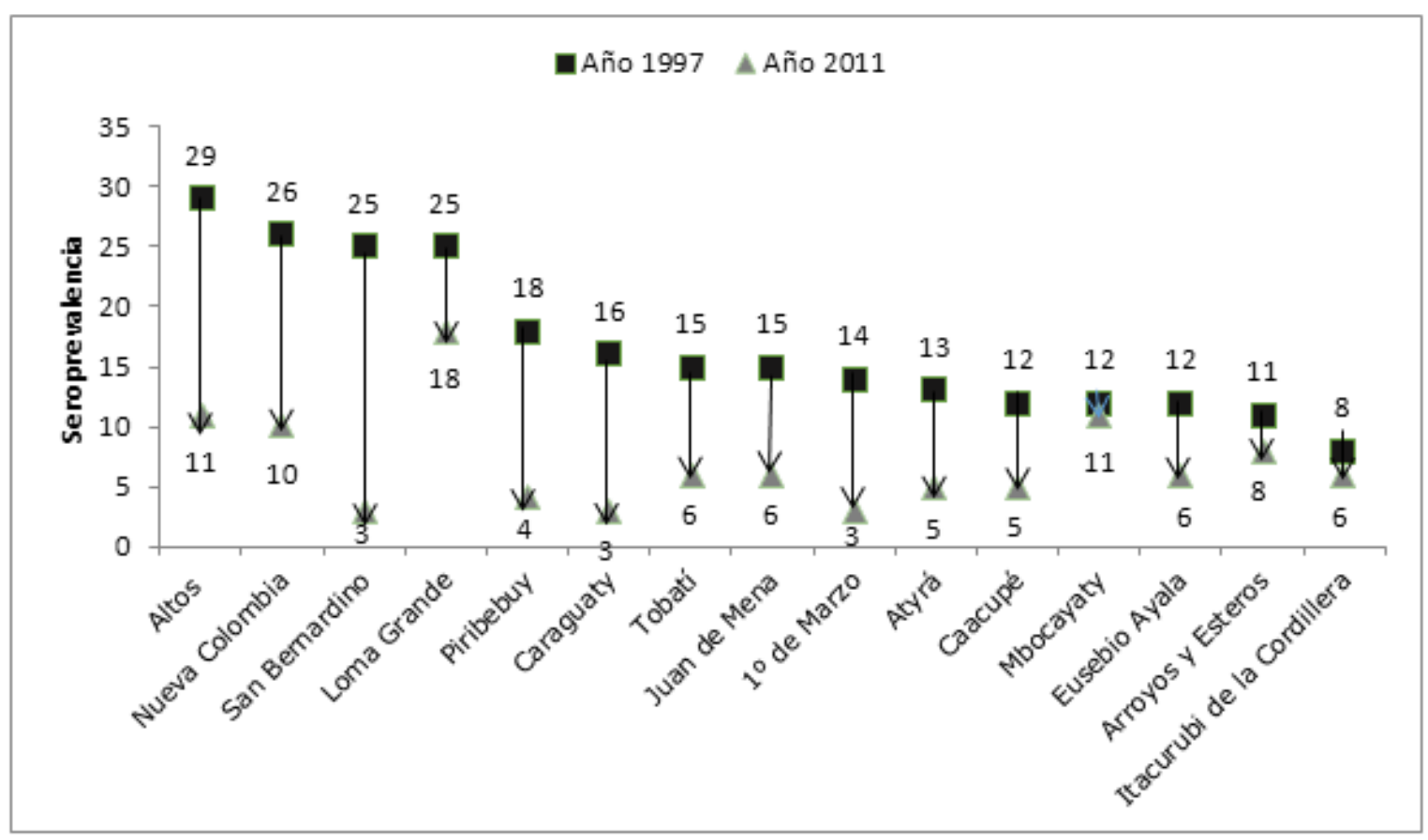

Fuente: Base de datos proveído por la III Región Sanitaria de Cordillera. Año 2012

Figura 2Seroprevalencia de la enfermedad de Chagas por distrito del departamento de cordillera en los años 1997 y 2011

\section{DISCUSIÓN}

En el presente estudio se pudo determinar la seroprevalencia de la enfermedad de Chagas en embarazadas del departamento de Cordillera en dos periodos diferenciados por la implementación del tamizaje de la infección en el control prenatal. Antes de la implementación en el año 1997, la seroprevalencia era del $15 \%$ y posterior a la implementación se redujo a $6 \%$ en el año 2011. Estos resultados son coincidentes con un estudio publicado en el año 2009 que incluyó embarazadas del mismo departamento que reveló una prevalencia de la enfermedad del 15\% en el año 1999 y el mismo estudio mostró la prevalencia de la enfermedad en el año 2009 del $6 \%$ (4).

Se destaca un importante descenso de la prevalencia de la enfermedad (9\%) en un periodo de 15 años. Según Russumando (2009), desde el año 1997 el programa prenatal está siendo localmente sustentable técnica y económicamente en Cordillera. Las estrategias implementadas en todos los servicios de Puestos, Centro de salud y Hospitales Regionales, fueron: a) el diagnóstico de la enfermedad de Chagas dentro del control prenatal ejecutado en forma descentralizada, equidistantes a los 20 distritos para que los mismos sean accesibles a toda la población de embarazadas; b) se adiestraron funcionarios entre bioquímicos y auxiliares de enfermería como laboratoristas de los Centros de Salud y de los Hospitales Regionales; estos encargados eran responsables de los estudios serológicos e interpretación de resultados para la detección de anticuerpos anti-T. cruzi por la técnica de ELISA, empleando un reactivo de producción local (Chagas Kit, IICS); c)se incorporó un sistema de control de calidad en cada laboratorio con monitoreos mensuales; d) los servicios de salud involucrados durante los tres primeros años (1996-1998) participaban cada tres meses de las reuniones de gestión en la III Región Sanitaria, estas actividades permitieron una activa retroalimentación (4), también las acciones de control vectorial iniciadas en 1999 en forma continua en el tiempo y contigua geográficamente en toda la región oriental del Paraguay y al tamizaje serológico en bancos de sangre que alcanzó el $100 \%$ de cobertura para el año 1995 para la enfermedad de Chagas $(4,13)$.

Cabe destacar que la disminución del número de muestras analizadas en los laboratorios de referencia en el año 2011 sería por el hecho de que toda mujer seropositiva detectada durante el prenatal no ingresa al tamizaje serológico en embarazos posteriores y se estima que en 15 años el sistema ha evaluado al $70 \%$ de las mujeres en edad fértil en el 
departamento de Cordillera (4). Además el departamento está declarado libre de transmisión domiciliar por Triatoma infestans desde el año 2008, según certificación de la Comisión Intergubernamental de la Iniciativa Sub regional del Cono Sur (INCOSUR) $(4,10)$.

Existen ciertos factores que están involucrados en una u otra manera en el descenso de la prevalencia de la enfermedad de Chagas en todos los distritos del departamento de Cordillera. Estos factores podrían ser:

1) El mejoramiento de las viviendas precarias con materiales de adobe y paja comúnmente empleados en las construcciones representan una desventaja por el hecho de que las vinchucas se alojan en las paredes de adobe y techos de paja donde colonizan y constituyen un riesgo para la transmisión vectorial de la enfermedad. Con relación a esto según el Censo del año 2002 en Cordillera existen poco más de 50.000 viviendas particulares ocupadas, con un promedio de 5 residentes en cada una de ellas. El acceso a los servicios básicos de la vivienda tuvo una evolución positiva en las últimas décadas, sobre todo entre los años 1992 y 2002. El mejoramiento de las viviendas disminuye el riesgo de transmisión vectorial, siendo ésta una de las formas más importantes de transmisión (17).

2) La participación comunitaria, desde el año 1999 se ha dado inicio al sistema de vigilancia en talleres de formación de líderes comunitarios encargados de la vigilancia entomológica, las escuelas participan en la detección de vinchucas en viviendas luego de los rociados y las municipalidades fueron nominadas como entes receptores de las denuncias y canalizadores de control post-rociado (16).

3) En cuanto a la educación, según informaciones del censo 2002 en el departamento de Cordillera en la población de 10 años y más de edad por alfabetismo y sexo, había una tasa del 7\% de analfabetas 17. En el año 2005 se publica un informe por la prensa que Paraguay tendría una tasa de analfabetismo del $8,4 \% 21$ y esto tuvo un descenso según publicación de la misma prensa en el año 2011 al 5,2\% (22).

4) El crecimiento económico del departamento en los últimos 10 años a través del turismo, artesanía, gastronomía y otros $(18,19,20)$.

El distrito de San Bernardino fue en el que se observó el mayor descenso de la seroprevalencia (22\%). Este distrito es el que muestra gran crecimiento económico ya que es principal centro veraniego del país y fue una de las primeras ciudades del país que tuvo luz eléctrica (18).

El distrito de Mbocayaty del Yhaguy fue el que mostró un menor descenso (1\%) en los años estudiados. Esto puede deberse a que este distrito de acuerdo a los datos proveídos por la Dirección General de Encuestas Estadísticas y Censo 2012, fue el que presento menor crecimiento económico, la cantidad de varones es mayor que la de mujeres, y su población es mayoritariamente rural $84 \%$ (23).

Los distritos de Valenzuela, Santa Elena, Isla Pucú, San José Obrero en el año 1997 tenían una prevalencia de infección chagásica de $8 \%, 16 \%, 15 \%$ y $12 \%$ respectivamente y en el 2011 el número de muestras procesadas fue muy bajo para establecer una prevalencia. El distrito de Emboscada en el año 1997 tenía una prevalencia de infección del $17 \%$ y en el año 2011 no proceso ninguna muestra razón por la cual no se puede conocer si hubo variaciones.

La información obtenida con este estudio contribuye al sistema de control prenatal de la enfermedad de Chagas en la III Región Sanitaria del departamento de Cordillera, porque demuestra la efectividad del sistema de diagnóstico prenatal, y en consecuencia se realiza un diagnóstico temprano de posible transmisión congénita, para realizar un tratamiento precoz de los casos de infección congénita y de esta manera asegurar el éxito del tratamiento. Además los datos de este estudio demuestran la efectividad de las estrategias de control implementadas por el Ministerio de Salud Pública y Bienestar Social.

\section{AGRADECIMIENTOS}

A la III Región Sanitaria del departamento de Cordillera por los datos proveídos para la realización de este estudio y a la Sra. Bienvenida Maldonado por su colaboración.

A la Dra. Alicia Duarte, tutora del trabajo de grado presentado con estos datos en el Instituto Superior Profesional Avanzado (ISPA), para la obtención del Título de Licenciatura en Laboratorio Clínico.

A la MSc. Zunilda Sánchez por la revisión y sugerencias para la realización del manuscrito. 


\section{REFERENCIAS BIBLIOGRAFICAS}

1. WHO. First WHO report on neglected tropical diseases: Working to overcome the global impact of neglected tropical diseases /Internet/.Geneva: WHO; 2010 /citado 10 mar 2012/. Disponible en: http://www.who.int/neglected_diseases/2010r eport/en/\#

2. Zambrano P. Protocolo de vigilancia en salud pública: Chagas. Colombia: Instituto Nacional de Salud, 2014.

3. Organización Panamericana de la Salud. Estimación cuantitativa de la enfermedad de Chagas en las Américas. Montevideo, Uruguay: OMS; 2006.

4. Russomando G.Transmisión congénita de la enfermedad de Chagas en el Paraguay. Mem. Inst.Cienc. Salud. 2009; 7(2):55-64.

5. Organización Panamericana de la Salud. Taller del Cono Sur sobre enfermedad de Chagas: Conceptualización de la vigilancia epidemiológica, Buenos Aires, Argentina, setiembre 2003. Montevideo: OPS; 2004. OPS/DPC/CD/290/04.

6. Manrique-Abril F, Ospina JM, Herrera G, Florez AC, Pavia PX, Montilla $M$, et al. Diagnóstico de enfermedad de Chagas en mujeres embarazadas y recién nacidos de Moniquirá y Miraflores, Boyacá, Colombia. Infectio. 2013; 17(1):28-34.

7. Pinto Dias JC. La Enfermedad de Chagas como reto para la salud pública latinoamericana. En: Guhl F, editor. Memorias Primer Taller Internacional sobre Control de la Enfermedad de Chagas. Curso de Diagnóstico, Manejo y Tratamiento de la Enfermedad de Chagas. VI. Reunión de la Iniciativa Andina para el Control de la Enfermedad de Chagas. Universidad de los Andes Bogotá, Colombia; 2 - 6 de mayo de 2005. Bogota: Universidad de los Andes; 2005. p. 10 .

8. Organización Mundial de la Salud. Control de la Enfermedad de Chagas. Segundo informe del Comité de Expertos de la OMS. Ginebra: OMS; 2002. Serie de InformesTécnicos; 905, p73.

9. Schmunis GA, Dias JCP. Health care reform, decentralization, prevention and control of vector-borne diseases.CadSaúde Pública. 2000; 16:117-23.

10. OPS. XVIIa. Reunión de la Comisión Intergubernamental (CI) de la Iniciativa Subregional Cono Sur de Eliminación de T. infestans y la Interrupción de la Transmisión Transfusional de la Tripanosomiasis Americana Santiago de Chile. Informe Técnico, (online). Santiago de Chile; 2010. (acceso: setiembre 2016). Disponible en: http://bvcenadim.digemid.minsa.gob.pe/files/ publicaciones/ponencias/XVIIa.pdf.

11.González-Brítez N. Dinámica poblacional de triatominos (Hemiptera- Reduviidae) relacionados con la transmisión de Trypanosomacruzi en Paraguay con énfasis en Triatoma sordida. Mem. Inst. Investig. Cienc. Salud, 2013; 11(2):105-111
12. Sánchez Z. Rol Potencial de T. sordida en la enfermedad de Chagas en Paraguay (Tesis de Maestría). Asunción: IICS, UNA, 2011.

13. Rojas de Arias A, Russomando G. El control de la enfermedad de Chagas en Paraguay. En: Silveira AC, Rojas de Arias A, Segura E, Guillén G, Russomando $G$, Schenone $H$, et al. Control de la enfermedad de Chagas en los países del Cono Sur de América: Historia de una iniciativa internacional. 1991/2001. OPS, 2002. p. 269-300.

14. Russomando G, Almiron M, Candia N, Franco $L$, Sánchez $Z$, de Guillen I. Implementación y evaluación de un sistema localmente sustentable de diagnóstico prenatal que permite detectar casos de transmisión congénita de la enfermedad de Chagas en zonas endémicas del Paraguay. RevSocBrasMedTrop. 2005; 38(2):49-54.

15. Marquez Roa NA, Lemir de Zelada MO, Molas AC. Frecuencia serológica de infección por Trypanosoma cruzi en donantes de sangre en el Paraguay entre los años 2006 y 2011. Mem. Inst. Investig. Cienc. Salud, 2013; 11(2): 26-31.

16. Russomando G. Programa nacional de control de la enfermedad de Chagas del Paraguay [Internet]. SENEPA, Ministerio de Salud Pública y Bienestar Social, 2002 [citado 10 mar 2012]. Disponible en: http://www.fac.org.ar/fec/chagas2/llave/md 2/md209/russo.htm

17. Dirección General de Estadística Encuesta y Censo. Atlas censal de Paraguay, año 2002. Paraguay. www.dgeec.gov.py/Publicaciones/Biblioteca /AtlasCordilleracenso.pdf

18. Distritos del Departamento de Cordillera, Atractivos de San Bernardino [Internet]. Bienvenido a Paraguay.com; acceso: octubre 2016]. Disponible en: http://www.bienvenidoaparaguay.com/ciud ades.php?xmlcity $=60 \#$ sthash.UWIWnzOz.d puf

19. Atyra, entre las diez ciudades más saludables [Internet]. ABC color. 201618 de noviembre; Sección suplemento Centinela. [acceso: setiembre 2016]. Disponible en:

http://www.abc.com.py/edicionimpresa/suplementos/centinela/atyraentre-las-diez-ciudades-mas-saludables1538803.html

20. Bareiro D. Altos celebra hoy su fiesta patronal y de fundación [Internet]. Ultima Hora.com. 200910 de agosto. Sección Metropolitana - Interior. [acceso: setiembre 2016]. Disponible en: http://www.ultimahora.com/altos-celebrahoy-su-fiesta-patronal-y-fundacionn245453.html

21. Los analfabetos paraguayos son una población en descenso [Internet]. ABC color. 20058 de setiembre; Sección Edición- Impresa-locales. [acceso: setiembre 2016]. Disponible en: 
http://www.abc.com.py/edicion-

impresa/locales/los-analfabetos-

paraguayos-son-una-poblacion-en-

descenso-854862.html

22. El $5,2 \%$ de la población paraguaya es analfabeta, según datos oficiales [Internet]. ABC color. 20118 de setiembre; Sección Edición- Impresa-locales. [acceso: setiembre 2016]. Disponible en: http://www.abc.com.py/edicionimpresa/locales/el-52-de-la-poblacionparaguaya-es-analfabeta-segun-datosoficiales-305382.html

23. Mbocayaty del Yhaguy, según datos oficiales [Internet]. Wikipedia, la enciclopedia libre. [acceso: octubre 2016]. Disponible en: https://es.wikipedia.org/wiki/Mbocayaty_de I_Yhaguy 